Preprint of the paper:

"A General Numerical Model for Grounding Analysis in Layered Soils" I. Colominas, J. Gómez-Calviño, F. Navarrina, M. Casteleiro (2002) Advances in Engineering Software.

http:/caminos.udc.es/gmni 


\title{
A GENERAL NUMERICAL MODEL FOR GROUNDING ANALYSIS IN LAYERED SOILS
}

\author{
I. Colominas, J. Gómez-Calviño, F. Navarrina, and M. Casteleiro \\ GMNI-Group of Numerical Methods in Engineering \\ Dpt. of Applied Mathematics, Civil Engineering School \\ Universidad de La Coruña, Campus de Elviña, 15192 SPAIN \\ colominas@iccp.udc.es
}

\begin{abstract}
Safety of persons, protection of equipment and continuity of power supply are the main objectives of the grounding system of a large electrical installation. For its accurate design, it is essential to determine the potential distribution on the earth surface and the equivalent resistance of the system. In this paper, we present a numerical approach based on the Boundary Element method for grounding analysis in layered soils. The feasibility of this formulation is discussed by means of its application to a real grounding system with different kinds of layered soil models.
\end{abstract}

\section{Introduction}

Since the early days of the industrial use of electricity, obtaining the potential distribution in large electrical installations when a fault current is derived into the soil through a grounding system has been a challenging problem. A "grounding" or "earthing" system comprises all the interconnected grounding facilities of an specific area of an electrical installation, being the "grounding grid" (or the "grounded electrode") the main element of these systems. Thus, the potential distribution on the earth surface is the most important parameter that it is necessary to know in order to design a safe grounding system.

In most of real electrical substations, the grounding grid consists of a mesh of interconnected cylindrical conductors, horizontally buried and supplemented by ground rods vertically thrusted in certain places of the substation site. Its main objective is to carry and dissipate electrical currents produced during fault conditions into the ground, in order to ensure that a person in the vicinity of the grounded installation is not exposed to a critical electrical shock. Furthermore, the power supply continuity and the equipment integrity must be preserved. To attain these goals, the apparent electrical resistance of the grounding system must be low enough to guarantee that fault currents dissipate mainly through the grounding electrode into the soil. Moreover, electrical potential values between close points on the earth surface (that can be connected by a person) must be kept under certain maximum safe limits (step, touch and mesh voltages) established in most of the guides and legal procedures of grounding system design $[1,2]$.

Since the sixties, several methods and procedures for the grounding analysis and design of electrical substations have been proposed. These methods are generally based on the professional practice, on semi-empirical works, on experimental data obtained from scale model tests, or on intuitive ideas. Doubtlessly, these contributions represented an important improvement in the grounding analysis area. However, some problems have been reported such as the large computational requirements, the unrealistic results obtained when the segmentation of conductors is increased, and the uncertainty in the margin of error $[1,3]$.

Equations governing the electrical current dissipation into the soil are well-known and can be stated from Maxwell's Electromagnetic Theory. Nevertheless, their application and resolution for the computing of grounding grids of large installations in practical cases present some difficulties. Obviously, no analytical solutions can be obtained for most of 
real problems. On the other hand, the characteristic geometry of grounding systems (a mesh of interconnected bare conductors with a relatively small ratio diameter-length) makes very difficult the use of numerical methods. Thus, the use of techniques commonly applied for solving boundary value problems, such as finite elements or finite differences, is extremely costly since the discretization of the domain (the ground excluding the electrode) is required. Therefore, obtaining sufficiently accurate results should imply unacceptable computing efforts in memory storage and CPU time.

For all these reasons, in the last years, the authors have proposed a numerical approach based on the transformation of the differential equations that govern the physical phenomena onto an equivalent boundary integral equation and the subsequent application of the Boundary Element Method. Generally speaking, boundary element approaches are based esentially in the same interpolation concepts (that is, the meshing of the domain and the use of shape functions) initially developed for the Finite Element Method [4]. The BEM has its same advantages concerning generality and flexibility in the solution of problems in engineering applications, but in contrast with finite elements, the BEM approximations are made only on the boundary of the domain $[5,6,7]$.

The boundary element formulation proposed for the authors for the analysis of grounding systems embedded in uniform soils models $[8,9]$ can be stated in the general framework of the symmetric Galerkin boundary element approaches $[10,11,12]$. Thus, the statement of a variational form based on a weighted-residual approach of the boundary integral equation and the selection of a Galerkin type weighting lead to a general symmetric formulation, from it is possible to derive specific numerical ones of high accuracy [9]. Furthermore, the development of this BEM approach has allowed to explain from a mathematical point of view the anomalous asymptotic behaviour of the clasical methods proposed for grounding analyis, and to identify rigorously the sources of error [13]. Finally, this boundary element approach has been implemented in a CAD application for earthing systems[14, 15] that allows to analyze real grounding installations in real-time using widespread personal computers.

In this paper, we present a generalization of the boundary element formulation for grounding grids embedded in layered soils on the basis of the numerical approach proposed for uniform soil models. Furthermore, an application to several practical cases by using the geometry of a real earthing system is presented.

\section{Mathematical Model of the Problem}

\subsection{Governing Equations}

As we have stated in the introduction, the equations that govern the dissipation of electrical currents into the ground through a grounded electrode can be derived from Maxwell's Electromagnetic Theory. Thus, restricting the analysis to the electrokinetic steady-state response and neglecting the inner resistivity of the earthing conductors (potential is assumed constant at every point of the grounding electrode surface), the 3D problem can be written as

$$
\begin{gathered}
\operatorname{div}(\boldsymbol{\sigma})=0, \quad \boldsymbol{\sigma}=-\boldsymbol{\gamma} \operatorname{grad}(V) \text { in } E \\
\boldsymbol{\sigma}^{t} \boldsymbol{n}_{E}=0 \text { in } \Gamma_{E} ; \quad V=V_{\Gamma} \text { in } \Gamma ; V \rightarrow 0, \text { if }|\boldsymbol{x}| \rightarrow \infty ;
\end{gathered}
$$

where $E$ is the earth, $\boldsymbol{\gamma}$ is its conductivity tensor, $\Gamma_{E}$ is the earth surface, $\boldsymbol{n}_{E}$ is its normal exterior unit field and $\Gamma$ is the electrode surface [9].

Therefore, when the grounded electrode attains a voltage $V_{\Gamma}$ (Ground Potential Rise, or GPR) relative to remote earth, the solution to problem (1) gives the potential $V$ and the current density $\boldsymbol{\sigma}$ at an arbitrary point $\boldsymbol{x}$. Then, it is possible to obtain the potential distribution on the earth surface (and consequently, the step, mesh and touch voltages of the earthing system), as much as the total surge current and the equivalent resistance by means of the current density $\boldsymbol{\sigma}$ on $\Gamma[1,2]$. On the other hand, since $V$ and $\boldsymbol{\sigma}$ are proportional to the GPR value, it will be used the normalized boundary condition $V_{\Gamma}=1$ from here on.

In many of the methods and theoretical procedures proposed for grounding analysis, the soil is commonly considered homogeneous and isotropic. Then, conductivity $\boldsymbol{\gamma}$ is substituted by an apparent scalar conductivity $\gamma$ that must be experimentally obtained[1]. Obviously, this hypothesis does not introduce significant errors if the soil is essentially uniform in all directions in the vicinity of the grounding grid[1], and this model can be used with loss of accuracy if the soil resistivity changes slightly with depth. Nevertheless, safety parameters involved in the grounding design can significantly vary if the soil electrical properties change through the substation site (for example, due to changes of the material nature, or the humidity of the soil). Hence, it seems advisable to develop more advanced models that could take into account variations of the soil conductivity in the surroundings of the electrical installation. 
It is obvious that taking into account all variations of soil conductivity would never be affordable, neither from the economical nor from the technical point of view. For this reason, a more practical soil model consists of assuming the soil stratified in a number of horizontal or vertical layers, defined by an appropriate thickness and an apparent scalar conductivity that must be experimentally obtained. In fact, it is widely accepted that two-layer soil models should be sufficient to obtain good and safe designs of grounding systems in most practical cases[1]. This paper is devoted to studying the application of numerical methods for grounding analysis in stratified soil models, specially in cases with two different horizontal or vertical layers (figures 1 and 2).

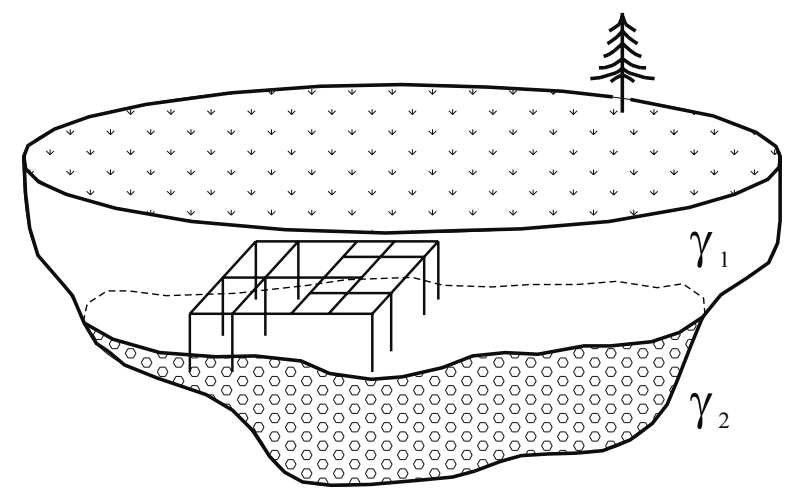

Figure 1. Scheme of a soil model with two horizontal layers.

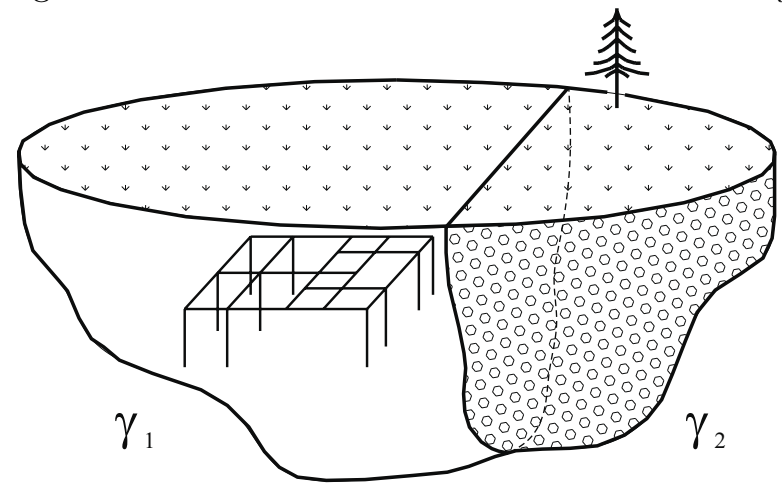

Figure 2. Scheme of a soil model with two vertical layers.

Therefore, in the hypothesis of a stratified soil model formed by $L$ layers with different conductivities, being the grounded electrode buried in the layer $b$, the mathematical problem (1) can be written in terms of the following Neumann exterior problem[18]

$$
\begin{gathered}
\operatorname{div}\left(\boldsymbol{\sigma}_{c}\right)=0, \boldsymbol{\sigma}_{c}=-\gamma_{c} \operatorname{grad}\left(V_{c}\right) \text { in } E_{c}, 1 \leq c \leq L ; \\
\quad \boldsymbol{\sigma}_{1}^{t} \boldsymbol{n}_{E}=0 \text { in } \Gamma_{E}, V_{b}=1 \text { in } \Gamma ; \\
V_{c} \rightarrow 0 \text { if }|\boldsymbol{x}| \rightarrow \infty, \boldsymbol{\sigma}_{c}^{t} \boldsymbol{n}_{c}=\boldsymbol{\sigma}_{c+1}^{t} \boldsymbol{n}_{c} \text { in } \Gamma_{c}, 1 \leq c \leq L-1 ;
\end{gathered}
$$

where $E_{c}$ is each one of the soil layers, $\gamma_{c}$ is its scalar conductivity, $V_{c}$ is the potential at an arbitrary point in the layer $E_{c}, \boldsymbol{\sigma}_{c}$ is its corresponding current density, $\Gamma_{c}$ is the interface between layers $E_{c}$ and $E_{c+1}$, and $\boldsymbol{n}_{c}$ is the normal field to $\Gamma_{c}[18]$.

\subsection{Integral Expression for Potential $V$}

Most of grounding systems of real electrical substations consist of a grid of interconnected bare cylindrical conductors, horizontally buried and supplemented by rods, being the ratio between the diameter and the length of the conductors relatively small $\left(\sim 10^{-3}\right)$. This apparently simple geometry implies serious difficulties in the modellization of the problem. Thus, it seems obvious that no analytical solutions can be obtained in most of real cases. On the other hand, the use of widespread numerical techniques (such as FEM or FDM) should involve a completely out of range computing effort, since it is required the discretization of the $3 \mathrm{D}$ domains $E_{c}$ (excluding the grounding electrode). For these reasons, we have turned our attention to other numerical techniques which require only the discretization of the boundaries. With this aim, it is essential to derive an integral expression for potential $V$ in terms of unknowns defined on the boundary $[7,9]$. 
During the construction process of the electrical installation, the surroundings of the substation site are levelled and regularized. Thus, the earth surface $\Gamma_{E}$ and the interfaces $\Gamma_{c}$ between layers can be assumed horizontal; consequently we will adopt an "horizontally layered soil model" in our mathematical model in order to catch the variations of the soil conductivity with depth (figure 1). In the case of variations of the soil conductivity near the grounding system, the simplest model we can state (a "vertically layered soil model") consist of asuming that the earth surface $\Gamma_{E}$ is horizontal, while the interfaces $\Gamma_{c}$ are parallel one to another and perpendicular to $\Gamma_{E}$ (figure 2).

Thus, taking into account these assumptions, the application of the "method of images" and Green's Identity to problem (2) yields the following integral expression $[16,17,18]$ for potential $V_{c}\left(\boldsymbol{x}_{c}\right)$ at an arbitrary point $\boldsymbol{x}_{c} \in E_{c}$, in terms of the unknown leakage current density $\sigma(\boldsymbol{\xi})\left(\sigma=\boldsymbol{\sigma}^{t} \boldsymbol{n}\right.$, where $\boldsymbol{n}$ is the normal exterior unit field to $\left.\Gamma\right)$ at any point $\boldsymbol{\xi}$ of the electrode surface $\Gamma \subset E_{b}$ :

$$
V_{c}\left(\boldsymbol{x}_{\boldsymbol{c}}\right)=\frac{1}{4 \pi \gamma_{b}} \iint_{\boldsymbol{\xi} \in \Gamma} k_{b c}\left(\boldsymbol{x}_{c}, \boldsymbol{\xi}\right) \sigma(\boldsymbol{\xi}) d \Gamma, \forall \boldsymbol{x}_{c} \in E_{c}
$$

That is, the potential at point $\boldsymbol{x}_{\boldsymbol{c}}$ due to a grounded electrode buried in the layer $b$ is the integral over all points $\boldsymbol{\xi}$ on the electrode surface $\Gamma$ of the leakage current density $\sigma$ that flows from each point $\boldsymbol{\xi} \in \Gamma$ weighted by the Green function $k_{b c}\left(\boldsymbol{x}_{c}, \boldsymbol{\xi}\right)$. These integral kernels are formed by series of infinite terms corresponding to the resultant images obtained when Neumann exterior problem (2) is transformed into a direct integral representation [7, 18]. Depending on the type of the soil model, these series can have an infinite or a finite number of terms [19]. In the case of uniform soil $(L=1)$, they are reduced to only two summands since there is only one image of the original grid $[8,9]$ :

$$
k_{11}\left(\boldsymbol{x}_{1}, \boldsymbol{\xi}\right)=\frac{1}{r\left(\boldsymbol{x}_{1},\left[\xi_{x}, \xi_{y}, \xi_{z}\right]\right)}+\frac{1}{r\left(\boldsymbol{x}_{1},\left[\xi_{x}, \xi_{y},-\xi_{z}\right]\right)}
$$

where $r\left(\boldsymbol{x}_{1},\left[\xi_{x}, \xi_{y}, \xi_{z}\right]\right)$ indicates the distance from $\boldsymbol{x}_{1}$ to $\boldsymbol{\xi} \equiv\left[\xi_{x}, \xi_{y}, \xi_{z}\right]$, being $\left[\xi_{x}, \xi_{y},-\xi_{z}\right]$ the symmetric point of $\boldsymbol{\xi}$ with respect to the earth surface $\Gamma_{E}$. We assume that the origin of the coordinates system is on the earth surface and the $z$-axis is perpendicular to $\Gamma_{E}$.

In the case of a vertical two-layered model (figure 2), the series of the kernels are also reduced to a finite number of terms:

$$
\begin{gathered}
k_{11}\left(\boldsymbol{x}_{1}, \boldsymbol{\xi}\right)=\frac{1}{r\left(\boldsymbol{x}_{1},\left[\xi_{x}, \xi_{y}, \xi_{z}\right]\right)}+\frac{1}{r\left(\boldsymbol{x}_{1},\left[\xi_{x}, \xi_{y},-\xi_{z}\right]\right)}+\frac{\kappa}{r\left(\boldsymbol{x}_{1},\left[\xi_{x},-\xi_{y}, \xi_{z}\right]\right)}+\frac{\kappa}{r\left(\boldsymbol{x}_{1},\left[\xi_{x},-\xi_{y},-\xi_{z}\right]\right)} \\
k_{12}\left(\boldsymbol{x}_{2}, \boldsymbol{\xi}\right)=\frac{1+\kappa}{r\left(\boldsymbol{x}_{2},\left[\xi_{x}, \xi_{y}, \xi_{z}\right]\right)}+\frac{1+\kappa}{r\left(\boldsymbol{x}_{2},\left[\xi_{x}, \xi_{y},-\xi_{z}\right]\right)}
\end{gathered}
$$

where $r\left(\boldsymbol{x},\left[\xi_{x}, \xi_{y}, \xi_{z}\right]\right)$ indicates the distance from $\boldsymbol{x}$ to $\boldsymbol{\xi}$. The other terms correspond to the distances from $\boldsymbol{x}$ to the symmetric point of $\boldsymbol{\xi}$ with respect to the earth surface $\Gamma_{E}$, and to the resultant images with respect to the vertical interface. We assume again that the origin of the coordinates system is on the earth surface and on the vertical interface, and the $z$-axis and $y$-axis are perpendicular to $\Gamma_{E}$ and $\Gamma_{1}$ respectively. Coefficient $\kappa$ is a ratio defined in terms of the layer conductivities

$$
\kappa=\frac{\gamma_{1}-\gamma_{2}}{\gamma_{1}+\gamma_{2}}
$$

In the case of an horizontal two-layered soil model (figure 1), the expressions of the integral kernels [17, 20] are given by

$$
\begin{aligned}
k_{11}\left(\boldsymbol{x}_{1}, \boldsymbol{\xi}\right)= & \sum_{i=0}^{\infty} \frac{\kappa^{i}}{r\left(\boldsymbol{x}_{1},\left[\xi_{x}, \xi_{y}, 2 i H+\xi_{z}\right]\right)}+\sum_{i=0}^{\infty} \frac{\kappa^{i}}{r\left(\boldsymbol{x}_{1},\left[\xi_{x}, \xi_{y}, 2 i H-\xi_{z}\right]\right)} \\
& +\sum_{i=1}^{\infty} \frac{\kappa^{i}}{r\left(\boldsymbol{x}_{1},\left[\xi_{x}, \xi_{y},-2 i H+\xi_{z}\right]\right)}+\sum_{i=1}^{\infty} \frac{\kappa^{i}}{r\left(\boldsymbol{x}_{1},\left[\xi_{x}, \xi_{y},-2 i H-\xi_{z}\right]\right)} \\
k_{12}\left(\boldsymbol{x}_{2}, \boldsymbol{\xi}\right)= & \sum_{i=0}^{\infty} \frac{(1+\kappa) \kappa^{i}}{r\left(\boldsymbol{x}_{2},\left[\xi_{x}, \xi_{y},-2 i H+\xi_{z}\right]\right)}+\sum_{i=0}^{\infty} \frac{(1+\kappa) \kappa^{i}}{r\left(\boldsymbol{x}_{2},\left[\xi_{x}, \xi_{y},-2 i H-\xi_{z}\right]\right)} \\
k_{21}\left(\boldsymbol{x}_{1}, \boldsymbol{\xi}\right)= & \sum_{i=0}^{\infty} \frac{(1-\kappa) \kappa^{i}}{r\left(\boldsymbol{x}_{1},\left[\xi_{x}, \xi_{y},-2 i H+\xi_{z}\right]\right)}+\sum_{i=0}^{\infty} \frac{(1-\kappa) \kappa^{i}}{r\left(\boldsymbol{x}_{1},\left[\xi_{x}, \xi_{y}, 2 i H-\xi_{z}\right]\right)} \\
k_{22}\left(\boldsymbol{x}_{2}, \boldsymbol{\xi}\right)= & \frac{1}{r\left(\boldsymbol{x}_{2},\left[\xi_{x}, \xi_{y}, \xi_{z}\right]\right)}-\frac{\left(1-\kappa^{2}\right) \kappa^{i}}{r\left(\boldsymbol{x}_{2},\left[\xi_{x}, \xi_{y}, 2 H+\xi_{z}\right]\right)}+\sum_{i=0}^{\infty} \frac{\left(\boldsymbol{x}_{2},\left[\xi_{x}, \xi_{y},-2 i H+\xi_{z}\right]\right)}{r .}
\end{aligned}
$$

In the above expressions, $r\left(\boldsymbol{x},\left[\xi_{x}, \xi_{y}, \xi_{z}\right]\right)$ indicates the distance from $\boldsymbol{x}$ to $\boldsymbol{\xi}$. The other terms correspond to the distances from $\boldsymbol{x}$ to the symmetric point of $\boldsymbol{\xi}$ with respect to the earth surface $\Gamma_{E}$, and to the interface surface between layers. $H$ is the thickness of the upper layer, and $\kappa$ is given by (6). We assume once more that the origin of the coordinates system is on the earth surface and the $z$-axis is perpendicular to $\Gamma_{E}$. 
As we can observe in expressions (4), (5) and (7), weakly singular kernels $k_{b c}\left(\boldsymbol{x}_{c}, \boldsymbol{\xi}\right)$ depend on the conductivity of the layers, and on the inverse of the distances from the point $\boldsymbol{x}_{c}$ to the point $\boldsymbol{\xi}$ and to all the images of $\boldsymbol{\xi}$ with respect to the earth surface $\Gamma_{E}$ and to the interfaces $\Gamma_{c}$ between layers [18, 19]. For uniform and two-layer soil models, these kernels can be expressed in the general form:

$$
k_{b c}\left(\boldsymbol{x}_{c}, \boldsymbol{\xi}\right)=\sum_{l=0}^{l_{k}} k_{b c}^{l}\left(\boldsymbol{x}_{c}, \boldsymbol{\xi}\right), \quad k_{b c}^{l}\left(\boldsymbol{x}_{c}, \boldsymbol{\xi}\right)=\frac{\psi^{l}(\kappa)}{r\left(\boldsymbol{x}_{c}, \boldsymbol{\xi}^{l}(\boldsymbol{\xi})\right)},
$$

where $\psi^{l}$ is a weighting coefficient that depends only on the ratio $\kappa$ defined by $(6)$, and $r\left(\boldsymbol{x}_{c}, \boldsymbol{\xi}^{l}(\boldsymbol{\xi})\right)$ is the Euclidean distance between the points $\boldsymbol{x}_{c}$ and $\boldsymbol{\xi}^{l}$, being $\boldsymbol{\xi}^{0}$ the point $\boldsymbol{\xi}$ on the electrode surface $\left(\boldsymbol{\xi}^{0}(\boldsymbol{\xi})=\boldsymbol{\xi}\right)$, and being $\boldsymbol{\xi}^{l}(l \neq 0)$ the images of $\boldsymbol{\xi}$ with respect to the earth surface and to the interfaces between layers [18]. Finally, $l_{k}$ is the number of summands in the series of integral kernels, and it depends on the case being analyzed (see (4), (5) and (7)).

\subsection{Variational Form of the Problem}

The integral expression for the potential (3) is also satisfied on the electrode surface $\Gamma[7,9]$, where the potential value is known by the boundary condition $V_{b}(\boldsymbol{\chi})=1, \forall \boldsymbol{\chi} \in \Gamma$. Consequently, the leakage current density $\sigma$ must verify the following Fredholm integral equation of the first kind on $\Gamma$

$$
\frac{1}{4 \pi \gamma_{b}} \iint_{\boldsymbol{\xi} \in \Gamma} k_{b b}(\boldsymbol{\chi}, \boldsymbol{\xi}) \sigma(\boldsymbol{\xi}) d \Gamma=1, \quad \forall \boldsymbol{\chi} \in \Gamma .
$$

It is important to remark that obtaining the leakage current density $\sigma$ from (9) is the key to solve the problem, since the potential distribution produced when a fault current is derived through the grounding grid can be easily obtained by means of expression (3) provided that the value of $\sigma$ is known. Furthermore, most of the remaining safety and design parameters (such as the equivalent resistance and the total surge current) can also be directly computed in terms of $\sigma$ $[9]$.

The above equation can be written in a variational form if one imposes that (9) is verified in the sense of weighted residuals, i.e., the following integral identity

$$
\iint_{\boldsymbol{\chi} \in \Gamma} w(\boldsymbol{\chi})\left(\frac{1}{4 \pi \gamma_{b}} \iint_{\boldsymbol{\xi} \in \Gamma} k_{b b}(\boldsymbol{\chi}, \boldsymbol{\xi}) \sigma(\boldsymbol{\xi}) d \Gamma-1\right) d \Gamma=0
$$

must hold for all members $w(\boldsymbol{\chi})$ of a suitable class of test functions defined on $\Gamma[8,9]$. Now, it is clear that a numerical approach based on the Boundary Element Method seems to be the best choice to solve equation (10) and to obtain the leakage current density $\sigma$, since its resolution only requires the discretization of the surface of the cylindrical conductors.

\section{BEM Numerical Formulation}

\subsection{General 2D approach}

The leakage current density $\sigma$ and the electrode surface $\Gamma$ can be discretized as

$$
\sigma(\boldsymbol{\xi})=\sum_{i=1}^{\mathcal{N}} \sigma_{i} N_{i}(\boldsymbol{\xi}), \quad \Gamma=\bigcup_{\alpha=1}^{\mathcal{M}} \Gamma^{\alpha}
$$

for a given set of $\mathcal{N}$ trial functions $\left\{N_{i}(\boldsymbol{\xi})\right\}$ defined on $\Gamma$ and a given set of $\mathcal{M}$ two dimensional boundary elements $\left\{\Gamma^{\alpha}\right\}$. Next, the integral expression (3) for the potential $V_{c}\left(\boldsymbol{x}_{c}\right)$ can also be discretized as

$$
\begin{gathered}
V_{c}\left(\boldsymbol{x}_{c}\right)=\sum_{i=1}^{\mathcal{N}} \sigma_{i} V_{c, i}\left(\boldsymbol{x}_{c}\right) ; \quad V_{c, i}\left(\boldsymbol{x}_{c}\right)=\sum_{\alpha=1}^{\mathcal{M}} \sum_{l=0}^{l_{V}} V_{c, i}^{\alpha l}\left(\boldsymbol{x}_{c}\right) \\
V_{c, i}^{\alpha l}\left(\boldsymbol{x}_{c}\right)=\frac{1}{4 \pi \gamma_{b}} \iint_{\boldsymbol{\xi} \in \Gamma^{\alpha}} k_{b c}^{l}\left(\boldsymbol{x}_{c}, \boldsymbol{\xi}\right) N_{i}(\boldsymbol{\xi}) d \Gamma^{\alpha}
\end{gathered}
$$

where $l_{V}$ represents the number of summands to consider in the evaluation of the series of kernels until convergence is achieved $\left(l_{V}=l_{k}\right.$ if this number is finite). 
On the other hand, for a given set of $\mathcal{N}$ test functions $\left\{w_{j}(\boldsymbol{\chi})\right\}$ defined on $\Gamma$, the variational form (10) is reduced to the linear system of equations:

$$
\begin{gathered}
\sum_{i=1}^{\mathcal{N}} R_{j i} \sigma_{i}=\nu_{j} \quad(j=1, \ldots, \mathcal{N}) \\
R_{j i}=\sum_{\beta=1}^{\mathcal{M}} \sum_{\alpha=1}^{\mathcal{M}} \sum_{l=0}^{l_{R}} R_{j i}^{\beta \alpha l}, \quad \nu_{j}=\sum_{\beta=1}^{\mathcal{M}} \nu_{j}^{\beta},
\end{gathered}
$$

being

$$
\begin{gathered}
R_{j i}^{\beta \alpha l}=\frac{1}{4 \pi \gamma_{b}} \iint_{\boldsymbol{\chi} \in \Gamma^{\beta}} w_{j}(\boldsymbol{\chi}) \iint_{\boldsymbol{\xi} \in \Gamma^{\alpha}} k_{b b}^{l}(\boldsymbol{\chi}, \boldsymbol{\xi}) N_{i}(\boldsymbol{\xi}) d \Gamma^{\alpha} d \Gamma^{\beta}, \\
\nu_{j}^{\beta}=\iint_{\boldsymbol{\chi} \in \Gamma^{\beta}} w_{j}(\boldsymbol{\chi}) d \Gamma^{\beta},
\end{gathered}
$$

where $l_{R}$ represents the number of summands to consider in the evaluation of the series of kernels until convergence is achieved $\left(l_{R}=l_{k}\right.$ if this number is finite).

Solution of the linear system (14) provides the values of the current densities $\sigma_{i}(i=1, \ldots, \mathcal{N})$ leaking from the nodes of the grid. However, the statement of this system requires the discretization of a $2 \mathrm{D}$ domain (the whole surface $\Gamma$ of the grounded electrodes). Moreover, the corresponding matrix is full and to compute its coefficients one must perform double integration on $2 \mathrm{D}$ domains. These facts imply a large number of degrees of freedom and an unaffordable computing requirements in the analysis of practical cases. For all these reasons, it is necessary to introduce some additional hypotheses in order to decrease the computational cost[9].

\subsection{Approximated BE approach and efficiency of the numerical scheme}

As we have previously mentioned, grounding grids of most electrical installations are formed by a mesh of conductors which ratio diameter/length is very small $\left(\sim 10^{-3}\right)$. Due to this specific geometry, the hypothesis of circumferential uniformity can be introduced[1,9]. In this way, the leakage current density $\sigma$ is assumed constant around the cross section of the cylindrical conductors of the grounding grid, and discretizations (11) and (14) become much simpler, since the classes of test and trial functions are restricted to those with circumferential uniformity while only the axial lines of the grounding electrodes have to be discretized [9]. Consequently, in comparison with the general 2D boundary element formulation, the number of elemental contributions $R_{j i}^{\beta \alpha}$ and $\nu_{j}^{\beta}$ needed to state the system of linear equations (14) and the number of unknowns $\sigma_{i}$ are now significantly smaller for a given level of mesh refinement.

In spite of the important reduction in the computational cost, extensive computing is still necessary mainly because of the circumferential integration on the perimeter of the electrodes that are involved in the integral kernels. In previous works we have proposed the approximated evaluation of these circumferential integrals by using specific quadratures[9]. The final result is an approximated 1D approach similar to the presented in section 3.1., where computing the coefficients of the equations system only requires integration on $1 \mathrm{D}$ domains, i.e. the axial lines of the electrodes[18].

Now, specific numerical approaches can be derived for different selections of the sets of trial and test functions (e.g., Point Collocation or Galerkin schemes). Further discussion in this paper is restricted to the case of a Galerkin type approach, where the matrix of coefficients is symmetric and positive definite[9]. The authors have derived a highly efficient analytical technique to evaluate the coefficients of the linear system of equations[9] for Point Collocation and Galerkin type weighting in uniform soil models. Since the $1 \mathrm{D}$ approximated expressions for the terms $V_{c, i}^{\alpha l}$ and $R_{j i}^{\beta \alpha l}$ in (13) and (15) are formally equivalent to those obtained in the case of uniform soil models, their computation can also be performed analytically by means of the above mentioned techniques [18].

This aspect of the numerical approach is the key for its generalization for any layered soils. Since the integral kernels are expressed in the general form given by (8), the coeficients of the stiffness matrix $R_{j i}$ are computed by the double sum in the $\mathcal{M}$ elements of the series of $l_{R}$ terms $R_{j i}^{\beta \alpha l}$ defined by (15). And these terms can be computed by using the high efficient analytical techniques developed for the uniform soil cases [9].

Of course, an important aspect of the proposed numerical formulation must be consider: the corresponding total computational cost. In our case, for a given specific discretization $(\mathcal{M}$ elements of $p$ nodes each, and a total number of $\mathcal{N}$ degrees of freedom), a linear system (14) of order $\mathcal{N}$ must be generated and solved. Matrix generation process requires $O\left(\mathcal{M}^{2} p^{2} / 2\right)$ operations, since $p^{2}$ series of contributions of type (10) have to be computed for every pair of elements, and approximately half of them are discarded because of symmetry. In uniform soil models these series are 
formed by only two terms. However, in two-layer models the series can have an infinite number of them. Therefore, a sufficient number of these terms must be numerically added up until a tolerance condition is fulfilled or an upper limit of summands is achieved. Consequently, matrix generation should be much more expensive in two-layer models.

On the other hand, since the matrix is symmetric but not sparse, the system solving process requires $O\left(\mathcal{N}^{3} / 3\right)$ operations if the resolution is carried out with a direct method.

Hence, most of the computing effort is devoted to matrix generation in small/medium problems, while linear system resolution should prevail in medium/large ones. In these cases, the use of direct methods for the linear system resolution is out of range. Therefore iterative or semiiterative techniques will be preferable [21]. The best results have been obtained by a diagonal Preconditioned Conjugate Gradient algorithm [22] with assembly of the global matrix [9]. This technique has turned out to be extremely efficient for solving large scale problems, with a very low computational cost in comparison with the corresponding to matrix generation. The reason for this remarkable efficency must be sought for in the theoretical convergence behaviour of the conjugate gradient method in relation with the condition number of the problem $[10,22]$.

Table 1. Balaidos II Grounding System: General Characteristics

\begin{tabular}{|lr|}
\hline \multicolumn{2}{|c|}{ Balaidos II Characteristics } \\
\hline Max. Grid Dimensions: & $61.1 \mathrm{~m} \times 79.1 \mathrm{~m}$ \\
Grid Depth: & $0.80 \mathrm{~m}$ \\
Number of Grid Electrodes: & 107 \\
Number of Vertical Rods: & 67 \\
Electrode Diameter: & $11.28 \mathrm{~mm}$ \\
Vertical Rod Diameter: & $14.00 \mathrm{~mm}$ \\
Vertical Rod Length: & $1.5 \mathrm{~m}$ \\
Ground Potential Rise (GPR): & $10 \mathrm{kV}$ \\
\hline
\end{tabular}

\section{$\wedge \mathrm{y}$}

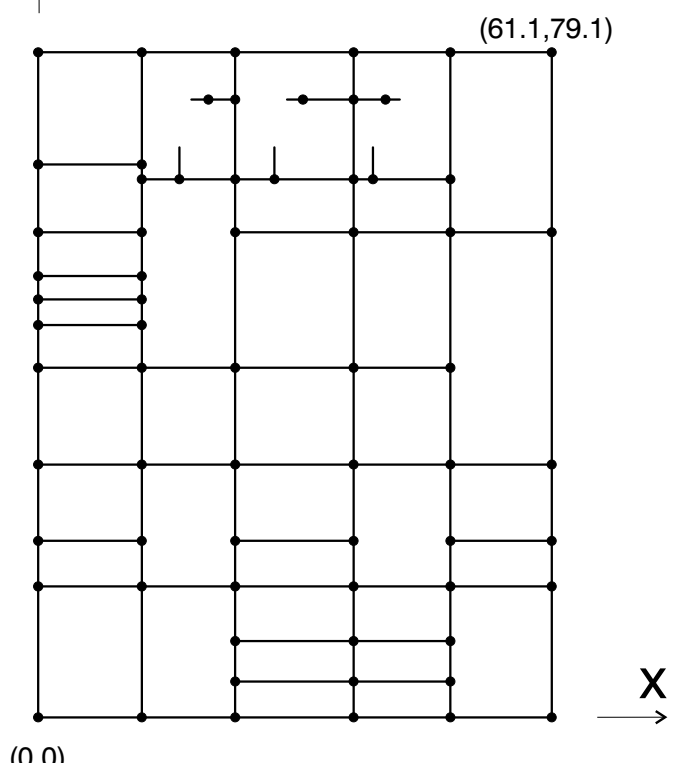

$(0,0)$

Figure 3. Balaidos II Grounding System: Plan of the grounding grid (vertical rods are marked with black points)

On the other hand, once the leakage current has been obtained, the cost of computing the equivalent resistance is negligible. The additional cost of computing potential at any given point (normally on the earth surface) by means of (12) only requires $O(\mathcal{M} p)$ operations, since $p$ contributions of type (13) have to be computed for every element. However, computing time may be important if it is necessary to compute potentials at a large number of points, for example to draw contour lines in a extense area of the grounding site.

The example presented in the next section corresponds to the analysis of a grounding system with different kind of soil models. We compare and discuss results obtained by using a uniform soil model and several two-layer soil models. 


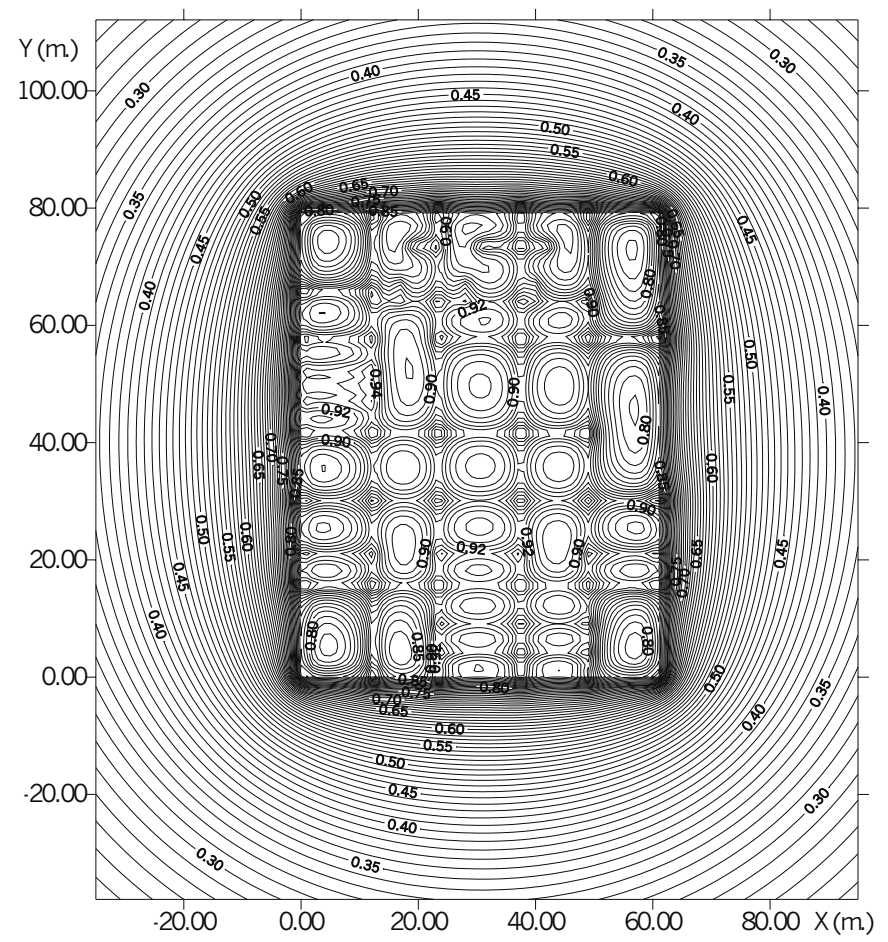

Figure 4. Balaidos II Grounding System: Potential distribution $(\times 10 \mathrm{kV})$ on the earth surface obtained by using a uniform soil model (model A).

\section{Application of the numerical approach to practical cases}

\subsection{Description of the earthing system}

The proposed numerical approach based on the Boundary Element Method has been implemented in a Computer Aided Design system for earthing analysis [14]. In this section we will present results obtained with different type of soil models by using the real geometry of a grounding grid: the Balaidos II substation, close to the city of Vigo in Spain.

The earthing system of this substation is a grid of 107 cylindrical conductors (diameter: $11.28 \mathrm{~mm}$ ) buried to a depth of $80 \mathrm{~cm}$, supplemented with 67 vertical rods (each one has a length of $1.5 \mathrm{~m}$ and a diameter of $14.0 \mathrm{~mm}$ ). The total surface protected is about $4800 \mathrm{~m}^{2}$. The total area studied is a rectangle of $150 \mathrm{~m}$ by $140 \mathrm{~m}$ (i.e., a surface about four times larger than the grounding grid). The Ground Potential Rise considered in this study has been $10 \mathrm{kV}$. The plan of the grounding grid is presented in figure 3 and a summary of the characteristics of the system is given in table 1 .

Table 2. Balaidos II Grounding System: Characteristics of the Numerical Model

\begin{tabular}{|lr|}
\hline \multicolumn{2}{|c|}{ BEM Numerical Model } \\
\hline Type of Approach: & Galerkin \\
Type of Current Density Element: & Linear \\
Number of Elements: & 241 \\
Degrees of Freedom: & 208 \\
\hline
\end{tabular}

\subsection{Results obtained by using different soil models}

The numerical model used in the resolution of this problem is based on a Galerkin type weighting. Each bar is discretized in one single linear leakage current density element, which implies a total of 208 degrees of freedom. In this case, the use of one single constant density element per electrode would imply a total of 241 , while the use of one single parabolic element would imply 449. Thus, linear density elements are not only the choice implying the least number of degrees of freedom in this case, but also the choice that offers the highest ratio between accuracy and computational cost. 


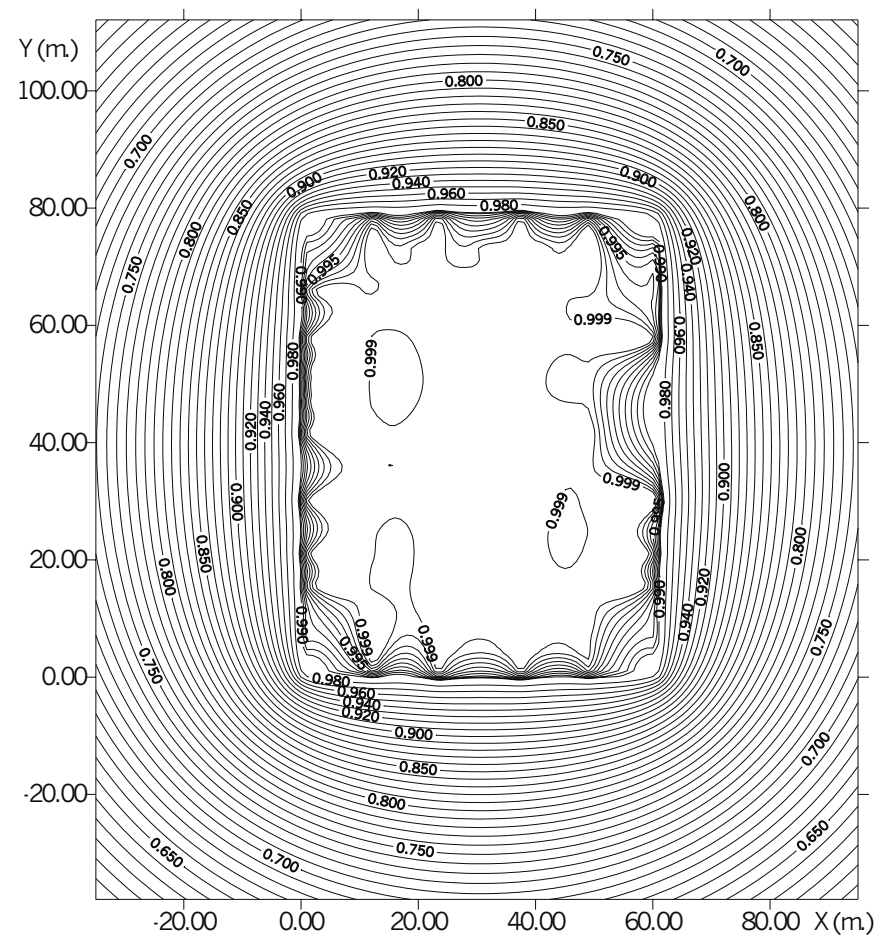

Figure 5. Balaidos II Grounding System: Potential distribution $(\times 10 \mathrm{kV})$ on the earth surface obtained by using an horizontal two-layer soil model (model B).

Table 3. Balaidos II Grounding System: Numerical results for the different soil models

\begin{tabular}{|l|r|r|r|}
\hline & \multicolumn{3}{|c|}{ Soil Models } \\
\cline { 2 - 4 } & $\mathrm{A}$ & $\mathrm{B}$ & $\mathrm{C}$ \\
\hline Equiv. Resistance $(\Omega)$ & 0.336 & 8.98 & 0.444 \\
Total Current $(\mathrm{kA})$ & 29.7 & 1.11 & 22.5 \\
CPU Time $(\mathrm{s})$ & 2.6 & 1093.5 & 4.2 \\
\hline
\end{tabular}

The examples presented have been repeatedly solved increasing the segmentation of the electrodes. At the scale of the whole grid, results and potential distributions on the earth surface were not noticeably improved by increasing segmentation. As a general rule, a reasonable level of segmentation is sufficient for practical purposes, and increasing the number of elements will not be necessary unless high accurate results must be obtained for a limited part of the whole earthing system. Furthermore, the use of higher order elements will be in general more advantageous than increasing segmentation intensively, since accuracy will be higher for a remarkably smaller total number of degrees of freedom. And consequently, it should require a considerably smaller computational effort [9].

In the Balaidos II grounding system, we have analyzed three different kind of soil models. "Model A" corresponds to a uniform soil model with $\gamma=50^{-1}(\Omega \mathrm{m})^{-1}$; "model B" corresponds to an horizontal two-layer soil model (as figure 1) with $\gamma_{1}=50^{-1}(\Omega \mathrm{m})^{-1}, \gamma_{2}=5000^{-1}(\Omega \mathrm{m})^{-1}$ and $H=1.5 \mathrm{~m}$; and "model C" corresponds to a vertical two-layer soil model (as figure 2) with $\gamma_{1}=50^{-1}(\Omega \mathrm{m})^{-1}, \gamma_{2}=5000^{-1}(\Omega \mathrm{m})^{-1}$ and the vertical interphase is located at a distance of $1 \mathrm{~m}$ from the grounding grid ( $\mathrm{y}=80.1 \mathrm{~m} \forall \mathrm{x}$ in the plan of figure 3 ).

Figure 4 shows the potential distribution on the earth surface obtained by using the uniform soil model A. Figures 5 and 6 show the potential distribution on the earth surface obtained by using the two-layer soil models B (horizontal) and $\mathrm{C}$ (vertical). Figure 7 is a comparison of the potential distribution on the earth surface obtained with models $\mathrm{A}$ and $\mathrm{C}$ in the zone where the conductivity of the ground varies.

Table 2 summarizes the characteristics of the numerical model, and Table 3 shows some of the results obtained in the three different cases: the equivalent resistance of the grounding system, the total current derived to the soil, and the CPU time.

The numerical approach has been implemented in a CAD system that runs in an Origin 2000 Silicon Graphics super- 


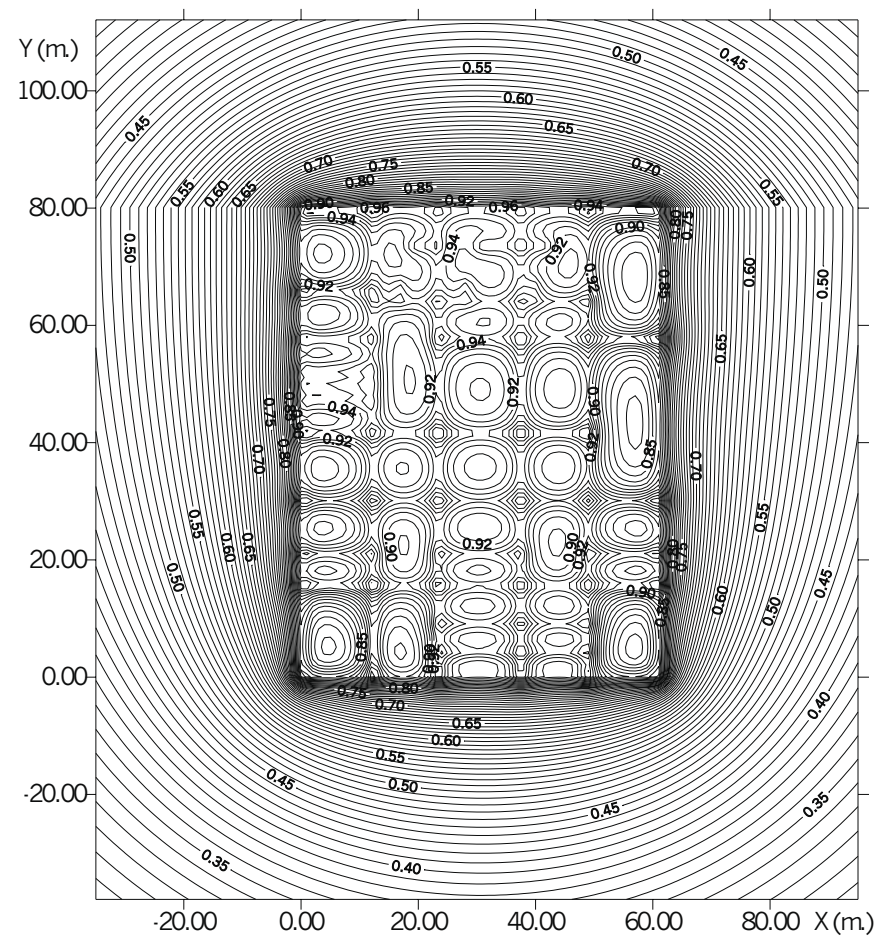

Figure 6. Balaidos II Grounding System: Potential distribution $(\times 10 \mathrm{kV})$ on the earth surface obtained by using a vertical two- layer soil model (model C).

computer at the European Center for Parallelism of Barcelona, CEPBA. Although the O2000 is a high-performance computer with 64 MIPS R10000 processors at $250 \mathrm{MHz}$, the CPU times given in Table 2 correspond to executing the examples in sequential mode, in order to compare the computational cost. It is clear that the computing time corresponding to model B is much lower when the code is executed in parallel mode, since the formulation accepts massive parallelization[23].

As we can see in these examples, the selection of a suitable soil model is essential to guarantee the safety in the grounding installations, since results obtained by using a multiple-layer soil model can be noticeably different from those obtained by using a single layer (or uniform) soil model (figures 4,5,6,7,8 and table 3). Therefore, it could be advisable to use multi-layer soil formulations to analyze grounding systems as a general rule, in spite of the increase of the computational effort. In fact, the use of this kind of advanced models should be mandatory in cases where the conductivity of the soil changes markedly with depth or in the vicinity of the substation site.

Obviously, this boundary element formulation can be applied to any other case with a higher number of layers. However, CPU time may increase up to unadmisible levels, mainly due to the poor rate of convergence of the underlying series expansions, and to the need of evaluating double series (in three-layer models), triple series (in four-layer models), and so on.

Nowadays, while single-layer models run in real time in conventional computers for the analysis of medium/big size grounding grids $[9,14]$, and two-layer models require a non negligible but still acceptable computing effort, multiplelayer models require in general an out of order computing time. For this reason, we are studying the improvement of the convergence speed of the series by using extrapolation techniques[24], and the use of acceleration techniques for slowly convergent series $[25,26]$. On the other hand, we are also working on the parallelization of the proposed approach, since this multi-layer BEM formulation could become a real-time design tool in a close future, as high-performance parallel computing becomes a widespread available resource in engineering[23].

\section{Conclusions}

In the last years, the authors have developed a highly efficient numerical formulation based on the Boundary Element method for earthing analysis in uniform soils, which has been succesfully applied to several real cases. 

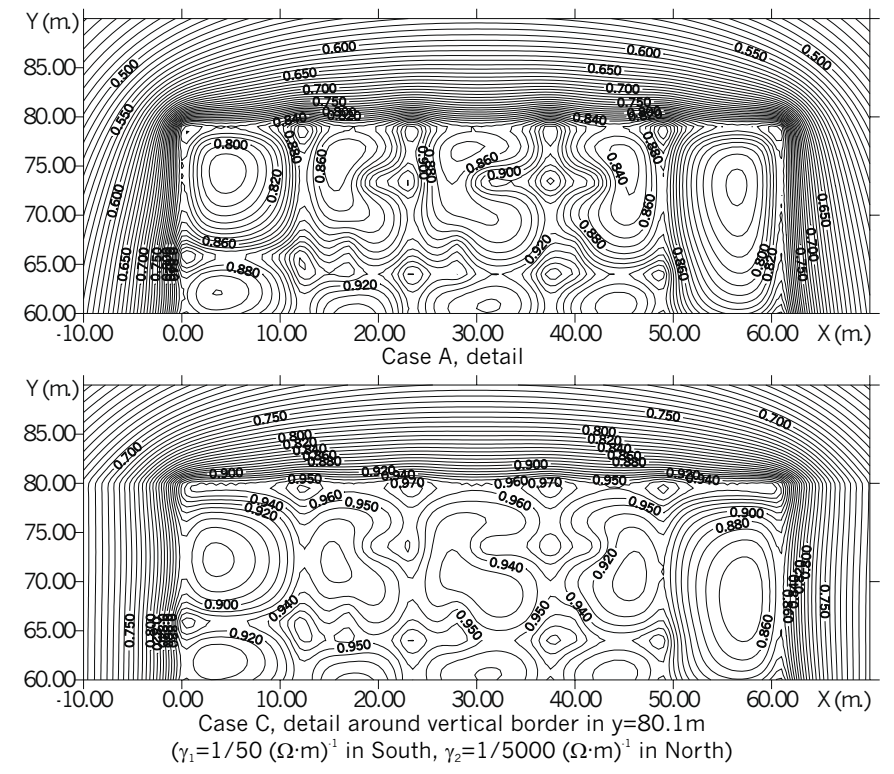

Figure 7. Balaidos II Grounding System: Comparison between the potential distribution $(\times 10 \mathrm{kV})$ on the earth surface obtained by using soil models $\mathrm{A}$ and $\mathrm{C}$ (detail of the grounding site close to the zone with change in the conductivity).

In this paper we have presented a generalization of this boundary element approach for grounding grids embedded in stratified soils. The proposed approach has been applied to the analysis of a real earthing system, considering different kinds of soil models: uniform soil, soil with two horizontal layers and soil with two vertical layers. The layered models allow to analyze the influence of the variations in the soil conductivity with depth and in the surroundings of the grounding system.

The suitable selection of a soil model is a key point in grounding analysis. We have shown that it is possible to obtain highly accurate results with the proposed boundary element numerical approach, and these results can be noticeably different depending on the kind of soil model considered in the study. Consequently, since the grounding safety parameters may significantly change, it could be advisable to use the multi-layer soil formulation as a general rule in spite of the increase in the computational cost.

\section{Acknowledgements}

This work has been partially supported by the "Ministerio de Ciencia y Tecnología (projects \#1FD97-0108 and \#DPI2001-0556)" of the Spanish Government cofinanced with FEDER funds, and by research fellowships of the "European Center for Parallelism of Barcelona, CEPBA", the "Xunta de Galicia" and the "Universidad de La Coruña".

\section{References}

[1] ANSI/IEEE Std.80, IEEE Guide for safety in AC substation grounding, New York, (2000).

[2] Sverak J.G., Progress in step and touch voltage equations of ANSI/IEEE Std.80, IEEE Trans. on Power Delivery,13, $762-767,(1999)$.

[3] Garret D.L., Pruitt J.G., Problems encountered with the APM of analyzing grounding systems, IEEE Trans. on Power Delivery,104, 4006-4023, (1985).

[4] Hughes T.J.R., The Finite Element Method, Prentice Hall, New Jersey, (1987).

[5] Brebbia C.A., Telles J.C.F., Wrobel L.C., Boundary element techniques, Springer Verlag, UK (1984).

[6] Banerjee P.K., The Boundary Element Method in engineering, Mc Graw-Hill, London (1995).

[7] Bonnet M., Boundary integral equation methods for solids and fluids, John Wiley \& Sons, Chichester, UK (1995). 


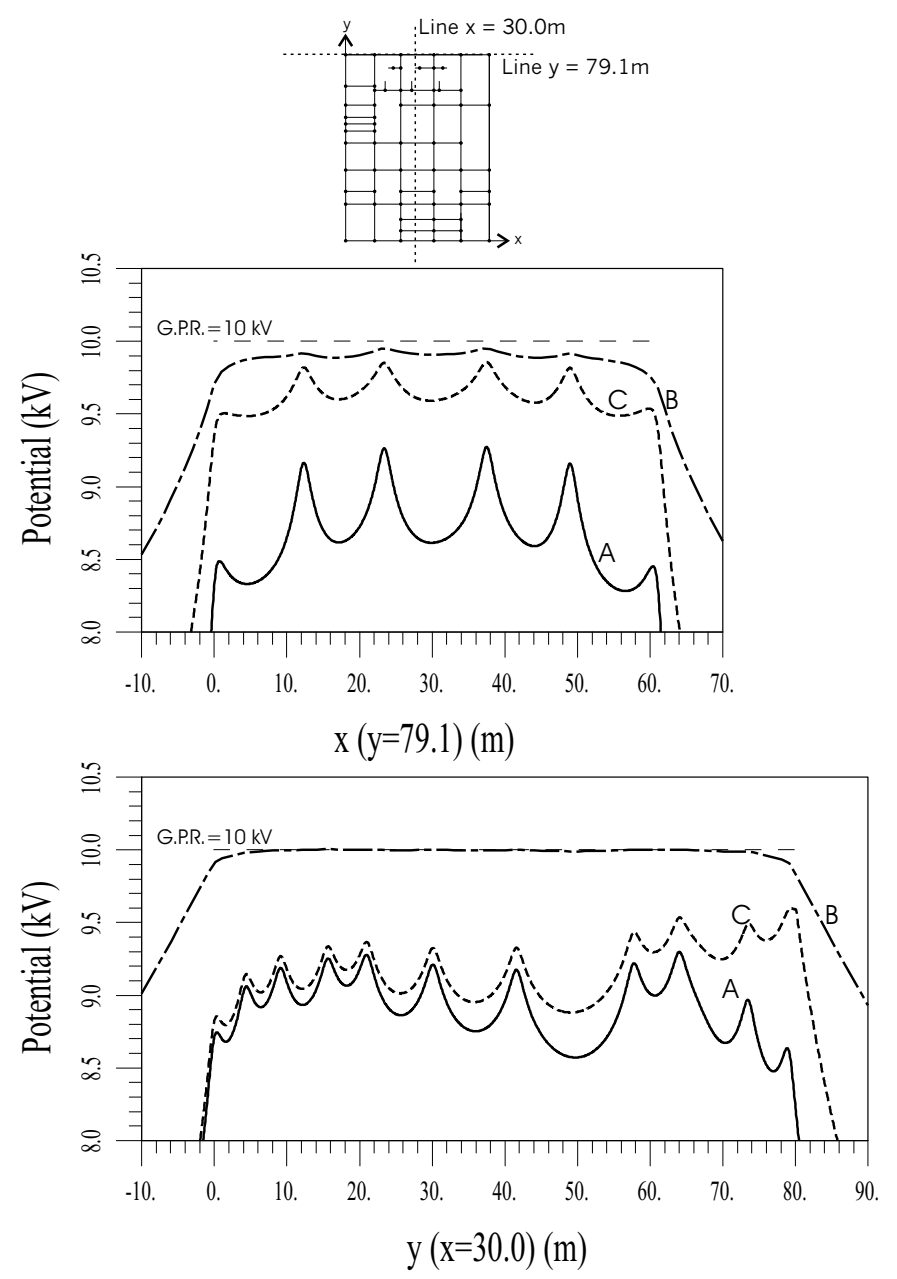

Figure 8. Balaidos II Grounding System: Potential profiles $(\mathrm{kV})$ on the earth surface along two lines for the three kinds of soil models. Notice the important differences in the results.

[8] Navarrina F., Colominas I., Casteleiro M., Analytical integration techniques for earthing grid computation by BEM, In "Numerical Methods in Engineering and Applied Sciences", 1197-1206, CIMNE, Barcelona, (1992).

[9] Colominas I., Navarrina F., Casteleiro M., A boundary element numerical approach for grounding grid computation, Comp. Meth. App. Mech. Engrg., 174, 73-90, (1999).

[10] Johnson C., Numerical solution of partial differential equations by the Finite Element Method, Cambridge Univ. Press, Cambridge, USA, (1987).

[11] Sirtori S., Maier G., Novati G., Miccoli S., A Galerkin symmetric boundary element method in elasticity: formulation and implementation, Int. J. Num. Meth. Engrg., 35, 255-282, (1992).

[12] Bonnet M., Maier G., Polizzotto C., On the symmetric Galerkin boundary element method, Appl. Mech. Rev., 51, 669-704, (1998).

[13] Navarrina F., Colominas I., Casteleiro M., Why do computer methods for grounding analysis produce anomalous results?, IEEE Trans. on Power Delivery, (2002). [Accepted for publication].

[14] Casteleiro M., Hernández L.A., Colominas I. and Navarrina F., Memory and User Guide of system TOTBEM for CAD of grounding grids in electrical installations, Civil Engineering School, Universidad de La Coruña, (1994).

[15] Colominas I., Navarrina F., Casteleiro M., A Boundary Element Formulation for the Substation Grounding Design, Adv. Engrg. Soft., 30, 603-700, (1999).

[16] Durand E., Électrostatique, Masson, Paris, (1966).

[17] Sunde E.D., Earth conduction effects in transmission systems, McMillan, New York, (1968). 
[18] Colominas I., Navarrina F., Casteleiro M., A numerical formulation for grounding analysis in stratified soils, IEEE Trans. on Power Delivery, (2001). [In press].

[19] Tagg G.F., Earth resistances, Pitman, New York, (1964).

[20] Dawalibi F.P., Mudhekar D. Optimum design of substation grounding in a two-layer earth structure, IEEE Trans. on Power Apparatus and Systems, 94, 252-272, (1975).

[21] Axelsson O., Iterative solution methods, Cambridge Univ. Press, Cambridge, USA, (1994).

[22] Saad Y., Iterative methods for sparse linear systems, University of Minnesota, USA, (2000).

[23] Colominas I., Navarrina F., Mosqueira G., Eiroa J.M., Casteleiro M., Numerical modelling for grounding grids in high-performace parallel computers, Boundary Elements XXII, WIT Press, Southampton, UK, (2000).

[24] Aneiros J.M., A numerical formulation for grounding analysis of electrical substations by using two-layer soil models, Ms.Sc. Thesys. Civil Engineering School, Universidad de La Coruña, (1996).

[25] Kinayman N., Aksun M.I., Comparative study of acceleration techniques for integrals and series in electromagnetic problems, Radio Science, 30, 1713-1722, (1995).

[26] Linton C.M., The Green's function for the two-dimensional Helmholtz equation in periodic domains, J. Engrg. Maths., 33, 377-402, (1998). 